\title{
Efecto de la adición de cáscara y semilla deshidratada en la capacidad antioxidante de una pasta de tomate producida en Sinaloa
}

\author{
Effect of the addition of husk and dehydrated seed on the antioxidant capacity of a tomato paste \\ produced in Sinaloa
}

\begin{abstract}
Nora Estela Ponce Fernández*, Gregorio Pollorena López, Cindy Rosas Domínguez, Sandra Carmina Osuna Izaguirre, Vida Mariel López Peñuelas

Instituto Tecnológico Superior de Guasave. Carretera Internacional Entronque a Brecha s/n, Ejido El Burrioncito, Guasave, Sinaloa, México. CP 81149.
\end{abstract}

\section{RESUMEN}

El objetivo fue evaluar el efecto de la adición de semilla y cáscara deshidratada en la capacidad antioxidante de pasta de tomate. Los tratamientos fueron: Pasta control (PC), adicionada con $1 \%$ (P1), $1.5 \%$ (P1.5) y $3 \%$ (P3) del subproducto seco $\left(70{ }^{\circ} \mathrm{C} / 6 \mathrm{~h}\right)$ (SCS). Se evaluó la capacidad antioxidante de 0 hasta la semana 24 de almacenamiento mediante determinación de fenoles totales, DPPH, TEAC y ORAC. EI diseño experimental fue ANOVA de una vía y las diferencias entre promedios se evaluaron mediante Tukey $(p \leq 0.05)$. El contenido de fenoles totales fue mayor en SCS (58.92 \pm 1.29 mgEAG/g) a los demás tratamientos ( $\mathrm{p} \leq 0.05)$, que aumentaron al adicionar el subproducto. La inhibición mayor del radical DPPH fue en SCS, seguido de P3 (67.207 $\pm 0.52 \mathrm{~g} / 100$ $\mathrm{g})$, con relación directa entre la adición del subproducto y la capacidad antioxidante en P1 y P1.5 ( $p>0.05$ ). La capacidad antioxidante de SCS y P3 en ORAC (53.69 \pm 0.41 y $21.52 \pm 3.45$ $\mu \mathrm{M} \mathrm{ET} / \mathrm{g})$ fue mayor que en TEAC $(14.72 \pm 0.50$ y $7.24 \pm 0.03 \mu \mathrm{M}$ $\mathrm{TE} / \mathrm{g})$, con la misma tendencia después de 24 semanas. Fenoles totales, DPPH, TEAC y ORAC disminuyeron respecto al tiempo. La adición de cáscara y semilla deshidratada modifica la capacidad antioxidante que se deteriora con el tiempo. Palabras clave: Pasta de tomate, capacidad antioxidante, cascara y semilla de tomate, subproducto

\section{ABSTRACT}

The objective was to evaluate the effect of the addition dehydrated husk and seed in antioxidant capacity of tomato paste. Control Paste (PC) added with $1 \%(\mathrm{P} 1), 1.5$ $\%$ (P1.5) and $3 \%$ (P3) of dried byproduct $\left(70{ }^{\circ} \mathrm{C} / 6 \mathrm{~h}\right)$ (SCS) were the treatments. Antioxidant capacity was evaluated at time 0 and week 24 of storage by total phenols, DPPH, TEAC and ORAC determination. The experimental design was one-way ANOVA and the differences between averages were evaluated by Tukey $(p \leq 0.05)$. SCS was the highest in total phenolic content (58.92 $11.29 \mathrm{mg}$ EAG/g), compared to the other treatments $(\mathrm{p} \leq 0.05)$, which increased when adding byproduct. SCS had higher DPPH inhibition, followed by P3 $(67.207 \pm 0.52 \%)$, with a direct relationship between the addition of the by-product and the antioxidant capacity of P1 and P1.5 ( $p>0.05)$. The antioxidant capacity of SCS and P3 in ORAC (53.69 \pm 0.41 and 21.52 $\pm 3.45 \mu \mathrm{M} \mathrm{TE} / \mathrm{g})$ was higher than in TEAC (14.72 \pm 0.50 and 7.24 $\pm 0.03 \mu \mathrm{M} \mathrm{TE} / \mathrm{g})$, with the same trend after 24 weeks of storage. Total phenols, DPPH, TEAC and ORAC decreased over time. The addition of dehydrated husk and seed modifies the antioxidant capacity that deteriorates over time.

Keywords: Tomato paste, antioxidant capacity, dehydrated husk and seed, byproduct.

\section{INTRODUCCIÓN}

El tomate rojo es un fruto que posicionó a Sinaloa como el productor número uno de México hasta 2017 y en 2018, fue el segundo producto más importante en valor de exportaciones agropecuarias mexicanas, con una participación de 12.8 por ciento. El tomate rojo (Solanum lycopersicum) es altamente consumido en fresco y procesado, ya que productos como enlatados y pastas de tomate brindan al consumidor la posibilidad de sustituir el fruto en épocas donde no está disponible (Büyükbay et al., 2009), aumentando vida de anaquel, aunado a que, estudios han demostrado fuertes correlaciones inversas entre el consumo de tomate y el riesgo de ciertos tipos de cáncer, enfermedades cardiovasculares y degeneración macular relacionada con la edad, ya que constituye una excelente fuente de compuestos como vitaminas $C$ y $E$, licopeno, $\beta$-caroteno, luteína y flavonoides como la quercetina (Dorais et al. 2008). Se sabe que el $80 \%$ del licopeno proviene del consumo de tomate crudo o de productos derivados. A pesar de los beneficios nutricionales del tomate, Vihna et al. (2014) demostraron que la eliminación de cáscara y la semilla, en la industria de procesamiento, tuvieron un efecto considerable sobre las propiedades fisicoquímicas, el contenido de compuestos bioactivos y la capacidad antioxidante de los frutos de tomate de cuatro cultivares típicos portugueses. El pelado causó una mayor disminución en el licopeno, el $\beta$-caroteno, el contenido de ácido ascórbico y fenólicos (promedios de $71 \%, 50 \%, 14 \%$ y $32 \%$, respectivamente) y redujeron significativamente la capacidad antioxidante de las frutas (8 \% y $10 \%$, utilizando ensayos de modelo DPPH y linoleato de $\beta$-caroteno, respectivamente). Aunque la eliminación de semillas favoreció el aumento tanto del color como de la dulzura, se perdieron algunos compuestos bioactivos (11\% de carotenoides y 24 $\%$ de fenólicos), así como la capacidad antioxidante (5 \%). De 
forma similar, Perea-Domínguez et al. (2018) demostraron que el subproducto del tomate es rico en compuestos fenólicos con alta actividad antioxidante, y sugirieron la valorización del subproducto industrial del tomate, que de acuerdo a la Planeación Agrícola Nacional 2017-2030 de SAGARPA, una de las estrategias es fomentar la industrialización del producto, lo que podría generar ingresos adicionales para la industria del tomate y reducir el problema de eliminación de desechos. Es por lo anterior, que el propósito de este artículo es evaluar el efecto de la adición de semilla y cáscara deshidratada en la capacidad antioxidante de una pasta de tomate producida en Sinaloa.

\section{MATERIALES Y MÉTODOS Preparación de la pasta de tomate}

Se utilizaron tomates saladette (Solanum lycopersicum L.), obtenidos en la región de Guasave, Sinaloa, en el ciclo 2018-2019. Se elaboraron cuatro formulaciones: pasta control (PC), pasta adicionada con $1 \%$ (P1), $1.5 \%$ (P1.5) y 3 $\%$ (P3) de subproducto de tomate compuesto por cáscara y semilla deshidratada en polvo (SCS). El tomate se desinfectó con $\mathrm{NaClO}$ al $0.01 \%$ por $5 \mathrm{~min}$, se escaldó a $90^{\circ} \mathrm{C}$ por $2 \mathrm{~min}$, se separó la pulpa de tomate (Pt), de la cáscara y semilla en la despulpadora (SCF) (DF600 3F, JAVAR), y se pesó. Para PC, la pulpa se evaporó en el módulo polivalente de procesamiento (De Lorenzo, DL Lab 50), con una alimentación de vapor a $110^{\circ} \mathrm{C}$ y $2 \mathrm{~atm}$ por tiempo entre 1 y 3 horas, al final se midieron los ${ }^{\circ}$ Brix. La semilla y cáscara se secó en un secador de bandejas a $70^{\circ} \mathrm{C}$ por $6 \mathrm{~h}$ y se pulverizó para agregarse a $\mathrm{P} 1$, P1.5 y P3, antes del proceso de evaporación. Posteriormente el producto envasado al vacío se etiquetó, pesó y almacenó a temperatura ambiente.

\section{Preparación de extracto hidrofílico}

Por triplicado se pesaron $10 \mathrm{~g}$ de las muestras húmedas: Pt (pulpa de tomate), PC, P1, P1.5, P3 y SCF (semilla y cáscara fresca); y $1 \mathrm{~g}$ de SCS (semilla y cáscara seca) en una balanza analítica PR 2003 Deltarange, se homogenizó cada muestra (Homogenizador IKA, ultra Turrax) en $20 \mathrm{~mL}$ de metanol al $80 \%$ (J.T. Baker), el homogenizado se colocó en un sonicador (Branson 2510) por 30 min y se centrifugó (SIGMA $3-30 \mathrm{KS})$ a $14,000 \mathrm{rpm}$ durante $15 \mathrm{~min}$ a $4{ }^{\circ} \mathrm{C}$. Se realizaron 2 lavados adicionales con $10 \mathrm{~mL}$ de metanol $80 \%$, colectando el sobrenadante para filtrarlo en papel Whatman No. 1. Finalmente los extractos obtenidos (Pt, PC, P1, P1.5, P3, SCF y SCS), se llevaron al mayor volumen resultante en muestras húmedas y en muestras secas con metanol $80 \%$ y se almacenaron a $-20^{\circ} \mathrm{C}$ hasta su uso en las determinaciones de fenoles totales y capacidad antioxidante (Shivashankara et al., 2004). El procedimiento se repitió a la semana 24.

\section{Contenido de Fenoles Totales}

El contenido de fenoles totales en los extractos hidrofilicos de tomate se determinaron de acuerdo al método de Folin-Ciocalteau (Singleton y Rossi, 1965) con algunas modificaciones. La determinación se realizó por triplicado. Se agregó $50 \mu \mathrm{L}$ de extracto, $3 \mathrm{~mL}$ de $\mathrm{H}_{2} \mathrm{O}$ (desionizada) y $250 \mu \mathrm{L}$ del reactivo de Folin-Ciocalteu $1 \mathrm{~N}$, cada muestra de agitó 8 minutos, y se adicionaron $750 \mu \mathrm{L}$ de $\mathrm{Na}_{2} \mathrm{CO}_{3}(20 \%)$ y $950 \mu \mathrm{L}$ de $\mathrm{H}_{2} \mathrm{O}$ (desionizada), las muestras se agitaron nuevamente y se incubaron por 30 min a temperatura ambiente. Después de la incubación, se leyó la absorbancia a 765 nm en un espectrofotómetro (UV-VIS HACH DR 6000). Los resultados fueron reportados como $\mathrm{mg}$ de equivalentes de ácido gálico (mg EAG) / g de peso seco. El procedimiento se repitió a la semana 24.

\section{DPPH (2,2-difenil-1-picril-hidracil)}

La capacidad de los extractos para inactivar al radical estable DPPH se evaluaron de acuerdo al método propuesto por Brand-Williams et al. (1995). Para la solución stock se mezclaron $2.5 \mathrm{mg}$ de radical DPPH con $100 \mathrm{~mL}$ de metanol puro. La solución se ajustó a una absorbancia de $0.7 \pm 0.02$ a $515 \mathrm{~nm}$. La reacción se llevó a cabo por triplicado donde se agregaron $3.9 \mathrm{~mL}$ de radical DPPH y $100 \mu \mathrm{L}$ del extracto. Las mezclas se agitaron durante 1 minuto y se dejaron reposar en la oscuridad durante 30 minutos. La reducción del radical se determinó a una absorbancia de $515 \mathrm{~nm}$ en un espectrofotómetro (UV-VIS HACH DR 6000). La actividad se expresó como porcentaje (\%) de inhibición del radical DPPH. El procedimiento se repitió a la semana 24 .

\section{TEAC (Capacidad Antioxidante en Equivalentes Trolox)}

El valor de TEAC se determinó a los PC, P1, P1.5, P3 y SCS después de 24 semanas de almacenamiento en condiciones normales, según la técnica de Pellegrini et al. (1999), que se basa en la habilidad de los extractos para inactivar el radical $\mathrm{ABTS}^{+}$(2,2'-azino-bis (3-ethylbenzthiazoline-6-sulfonic acid)). El radical catión se generó con $19.2 \mathrm{mg}$ de $\mathrm{ABTS}^{+}$ disueltos en $5 \mathrm{~mL}$ de agua desionizada y $88 \mu \mathrm{L}$ de $\mathrm{K}_{2} \mathrm{~S}_{2} \mathrm{O}_{8}$ $\left(0.0378 \mathrm{~g} \mathrm{~mL}^{-1}\right)$. La solución madre se incubó en la oscuridad a temperatura ambiente por $16 \mathrm{~h}$. Posteriormente, se tomó 1 $\mathrm{mL}$ de radical $\mathrm{ABTS}{ }^{+}$activado, se agregó a $88 \mathrm{~mL}$ de metanol y se ajustó a $0.7 \pm 0.02(734 \mathrm{~nm})$. Se adicionaron $2970 \mu \mathrm{L}$ de $\mathrm{ABTS}^{+}$a $30 \mu \mathrm{L}$ de cada extracto, se monitoreó la absorbancia a $734 \mathrm{~nm}$ al minuto 1 y 5 después del mezclado inicial. Los resultados se reportaron como $\mu$ moles de equivalentes Trolox $(\mu \mathrm{MET})$ / $\mathrm{g}$ de peso seco.

\section{ORAC (Capacidad de absorción de radicales de oxígeno)}

Para Capacidad atrapadora de radicales de oxígeno (ORAC) se enviaron las muestras (SCS, PC, P1, P1.5 y P3) a un laboratorio externo (CIIDIR IPN Unidad Guasave) para que realizaran los análisis después de 24 semanas de almacenamiento, siguiendo la metodología descrita por Prior et al. (2003) con modificaciones de Perea-Domínguez et al. (2018). Los extractos previamente disueltos en metanol, se agregaron en el buffer de ORAC (buffer de fosfato de potasio, $\mathrm{pH}$ 7.4) y se preparó una curva estándar Trolox (0-30 mg / L). La solución con $87 \mu \mathrm{M}$ fluoresceína y $70 \mathrm{mM}$ de AAPH $(2,20$-Azobis (2-amidinopropano) dihi-drocloruro)) se mezcló en el buffer fosfato. Después en una microplaca negra de 96 pozos 
con $25 \mu \mathrm{L}$ del blanco (buffer fosfato), control o muestra y se añadieron $200 \mu \mathrm{L}$ de solución de fluoresceína. Despues se añadió $50 \mu \mathrm{L}$ de AAPH recién preparada y se incubó a 37 ${ }^{\circ} \mathrm{C}$. La fluorescencia se registró cada 2 min durante 76 ciclos (longitud de onda de excitación de $485 \mathrm{~nm}$ y longitud de onda de emisión de $530 \mathrm{~nm}$ ) con un DTX 880 detector multimodo, Beckman Coulter (Brea, CA, EE. UU.) Los resultados se reportaron como $\mu$ moles de equivalentes Trolox ( $\mu \mathrm{M} E \mathrm{ET}) / \mathrm{g}$ de peso seco. Las determinaciones se hicieron por triplicado.

\section{Análisis estadístico}

El diseño experimental fue totalmente aleatorizado y los tratamientos fueron Pt, PC, P1, P1.5, P3, SCF y SCS por triplicado y los datos se evaluaron mediante un ANOVA bajo un diseño al azar de una sola vía, a través de MINITAB 16. Las diferencias entre las medias se evaluaron mediante la prueba de Tukey con un nivel de confianza de $95 \%$. Se establecieron coeficientes de correlación de Pearson entre los compuestos fenólicos y la capacidad antioxidante.

\section{RESULTADOS Y DISCUSIÓN}

Los compuestos fenólicos tienen la facilidad de captar radicales libres lo que les aporta actividad antioxidante y esto es debido a sus características estructurales. La figura 1 muestra el contenido de fenoles totales de los extractos de los tratamientos: Extracto pasta control (PC), extracto de pasta adicionado con $1 \%$ de semilla y cáscara en polvo (P1), extracto de pasta adicionado con $1.5 \%$ (P1.5), extracto de pasta adicionado con $3 \%$ (P3), extracto de pulpa de tomate $(\mathrm{Pt})$, extracto semilla y cáscara fresca (SCF) y, extracto de semilla y cáscara seca (SCS). En figura 1, A representa la medición en la semana 0 de procesamiento y $B$ los resultados después de 24 semanas de almacenamiento en bolsas al vacío y trasparentes.

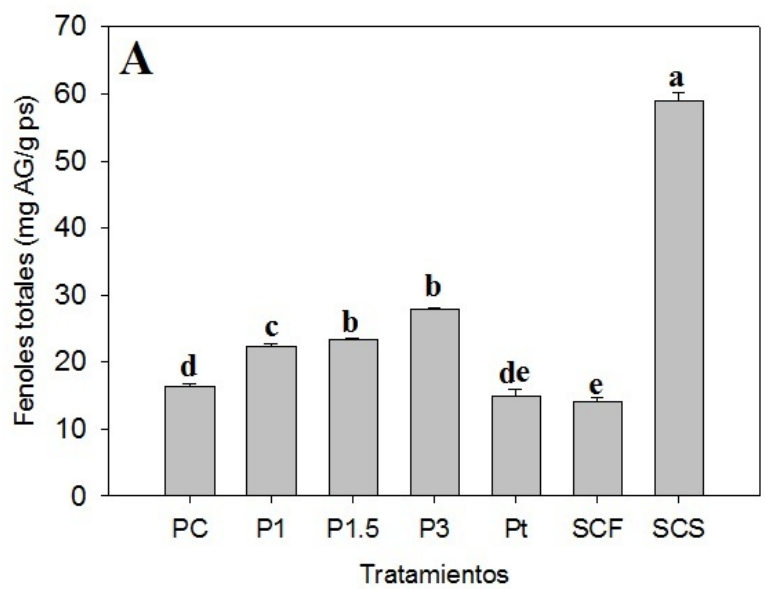

El contenido de fenoles totales en $A$, mostró que las muestras frescas ( $\mathrm{Pt}=$ pulpa de tomate; $\mathrm{SCF}=$ semilla y cascara fresca), se comportaron de forma similar a los valores 14.9960 $\pm 0.9010 \mathrm{mg}$ EAG / g y $14.1630 \pm 0.6310 \mathrm{mg}$ EAG / g obtenidos por Zhang et al. (2011), quienes mencionaron que en el escaldado, los compuestos fenólicos disminuyeron debido a un proceso de lixiviación; derivado de la destrucción de las paredes celulares y los compartimentos subcelulares (vacuolas y apoplastos) durante la ebullición facilitando la migración de sustancias hidrosolubles hacia el espacio extracelular y de allí al agua de procesamiento. El contenido fenólico fue mayor en SCS (58.92 / g \pm 1.29 mg EAG) con diferencia significativa a los demás tratamientos $(p \leq 0.05)$, menor a lo reportado por Perea-Domínguez, (2018), quienes analizaron los subproductos de tomate en muestras liofilizadas. Respecto a los tratamientos donde se adicionó cáscara y semilla deshidratada, se encontró una relación directa entre el porcentaje de adición y la concentración de fenoles, lo cual se atribuyó a lo mencionado por Vihna et al. (2014), quienes afirman que al retirar la cáscara y semilla se presenta un efecto negativo en la cantidad de compuestos bioactivos y capacidad antioxidante. Existen diferentes factores que degradan el contenido de polifenoles en los alimentos a través del tiempo. Vallverdú-Queralt et al. (2012) mencionaron que enzimas como la peroxidasa se involucran en la degradación oxidativa de compuestos fenólicos que interactúan con especies reactivas de oxígeno, como ocurrió en los tratamientos después de 24 semanas de almacenamiento (Figura 1.B), donde el tratamiento SCS ( $2.501 \pm 0.27 \mathrm{mg}$ EAG / g) presentó el contenido mayor en fenoles totales con diferencia significativa $(p \leq 0.05)$ a los demás tratamientos, resultado ligeramente mayor a lo reportado por Valdés-Morales et al. (2014) para polvo de cáscara y semilla de tomate saladette y AbouelYazeed et al. (2019) en polvo de semilla y cáscara de tomate,

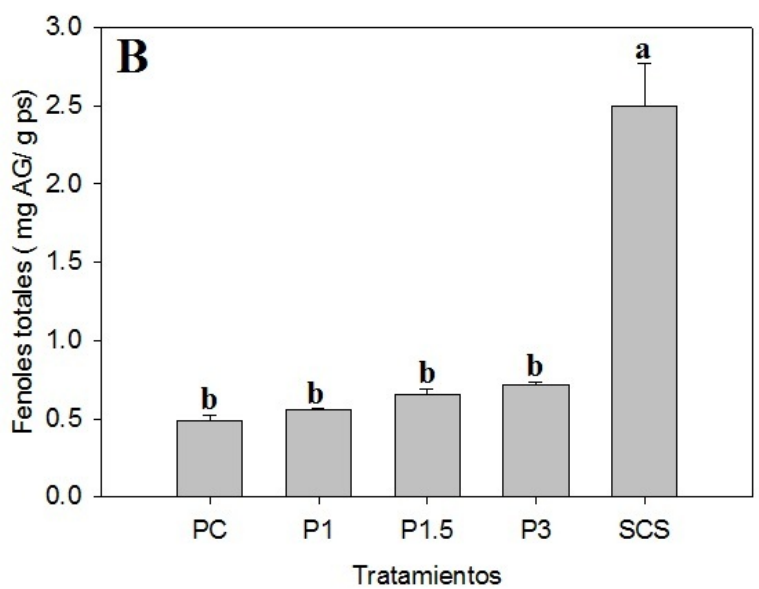

Figura 1. Resultados de Fenoles totales. A: Semana 0 de procesamiento; B: Semana 24 de almacenamiento. Diferente literal (abcde) entre columnas indica diferencia significativa (Tukey $\mathrm{p} \leq 0.05$ ). $\mathrm{PC}=$ Extracto pasta control; $\mathrm{P} 1=$ Extracto de pasta adicionado con $1 \%$ de semilla y cáscara en polvo; P1.5= Extracto de pasta adicionado con 1.5\%; P3= Extracto de pasta adicionado con $3 \%$; Pt= Extracto de pulpa de tomate; $\mathrm{SCF}=$ Extracto de semilla y cáscara fresca; $\mathrm{SCS}=$ Extracto de semilla y cáscara seca.

Figure 1. Results of total phenols. A: Week 0 of processing; B: Week 24 storage. abcde, different literal means statistically significant difference between treatments (Tukey $\mathrm{P} \leq 0.05$ ). $\mathrm{PC}=$ Control paste extract; $\mathrm{P} 1=$ Pasta extract added with $1 \%$ seed and shell powder; $\mathrm{P} 1.5=$ Pasta extract added with 1.5\%; P3 = Pasta extract added with $3 \% ; \mathrm{Pt}=$ Tomato pulp extract; SCF = Fresh seed and shell extract; SCS $=$ Seed extract and dried husk. 
donde se observó un deterioro de los compuestos fenólicos. A pesar del deterioro a través del tiempo, la tendencia de los resultados permaneció constante, entre los tratamientos referentes a pastas de tomate, el mayor contenido de fenoles fue en $\mathrm{P} 3$ sin diferencia significativa $(p>0.05)$ con P1 $(0.556 \pm$ $0.02 \mathrm{mg}$ EAG / g) y P1.5 (0.656 $\pm 0.04 \mathrm{mg} \mathrm{EAG} \mathrm{/} \mathrm{g),} \mathrm{sin} \mathrm{embar-}$ go, sí hubo diferencia con PC $(0.488 \pm 0.03 \mathrm{mg} E A G / g)$ lo que se atribuyó al contenido fenólico que aportó la adición de la semilla y cáscara de tomate, resultado menor a los mostrado por Vallverdú-Queralt et al. (2012), debido principalmente al procesamiento de las muestras por liofilización para la obtención de los extractos diferente al secado convencional.

Se sabe que la capacidad antioxidante total de tomate está dada por la suma de la actividad que aporte cada uno de los componentes bioactivos (carotenos (licopeno y $\beta$-caroteno), ácido ascórbico, tocoferol y compuestos fenólicos). Sin embargo, los más resistentes a los procesamientos térmicos son el licopeno y los compuestos fenólicos (Navarro-González et al. 2001). Según Valdez- Morales et al. (2014), los compuestos fenólicos más abundantes en la cáscara del tomate saladette son el ácido cafeico, ferúlico y vanílico; y, en la semilla son el ácido sinápico, ferúlico y vanílico, los cuales permanecieron activos después del tratamiento térmico.

Otro indicador importante, en la capacidad antioxidante es la inhibición del radical DPPH, que consiste en evaluar el potencial antioxidante de un compuesto, ya que es una técnica rápida, simple y sus resultados son comparables con otros métodos (Kedare y Singh, 2011). Los resultados (figura 2) mostraron mayor inhibición del radical DPPH en SCS (72.012 $\pm 2.664 \%)$ con diferencia significativa a los demás tratamientos $(\mathrm{p} \leq 0.05)$, dichos valores son mayores a lo reportado por Sarkar y Kaul (2014) en semilla y cáscara liofilizadas ( $37.5 \pm 1 \%$ ) y deshidratadas de forma convencional ( $34 \pm 0.8 \%)$, y a su vez, mayores a los encontrados por Valdés-
Morales et al. (2014) en subproductos de tomate liofilizados y a Elbadrawy y Sello (2016) en extractos de cáscara de tomate.

Con respecto a las pastas de tomate, P3 (67.207 \pm $0.52 \%)$, que contiene la mayor cantidad de cáscara y semilla deshidratada, no presentó diferencia significativa $(p>0.05)$ con P1 (64.228 $1.19 \%)$ y P1.5 (66.174 $\pm 0.461 \%)$, contrario a lo sucedido con PC, a pesar de que el porcentaje añadido varía del 1 al $3 \%$.

El almacenamiento prolongado en condiciones normales (Figura 2.B), provocó una disminución de la capacidad antioxidante similar a Vallverdú-Queralt et al. (2011), quienes evaluaron el porcentaje de inhibición a través del tiempo en productos derivados del tomate almacenados en condiciones controladas a $4{ }^{\circ} \mathrm{C} \pm 1$ y envases Tetrapak. Lo anterior, se atribuye a que la disminución más significativa de la capacidad antioxidante por almacenamiento ocurre entre 0 y 6 meses de almacenamiento.

TEAC es un método que se basa en la habilidad para inactivar el radical ABTS para evaluar la capacidad antioxidante de alimentos y bebidas, y ORAC es un método que permite medir la capacidad de absorción de radicales de oxígeno (Zulueta et al., 2009). Los resultados después de 24 semanas de almacenamiento en condiciones normales (Tabla 1), mostraron una tendencia similar a lo encontrado en fenoles y DPPH en la semana 0 de almacenamiento. Los resultados mostraron variación entre cantidades en función del método y el radical utilizado, a pesar de que son diferentes mecanismos de inactivación (reacción de transferencia de átomos de hidrógeno y métodos basados en la transferencia de electrones), estos presentan el mismo comportamiento (Karadag et al., 2009).

El valor más elevado de TEAC lo presentó SCS con diferencias significativa $(p \leq 0.05)$ con los demás tratamientos. Con respecto a las pastas de tomate, PC no presentó dife-
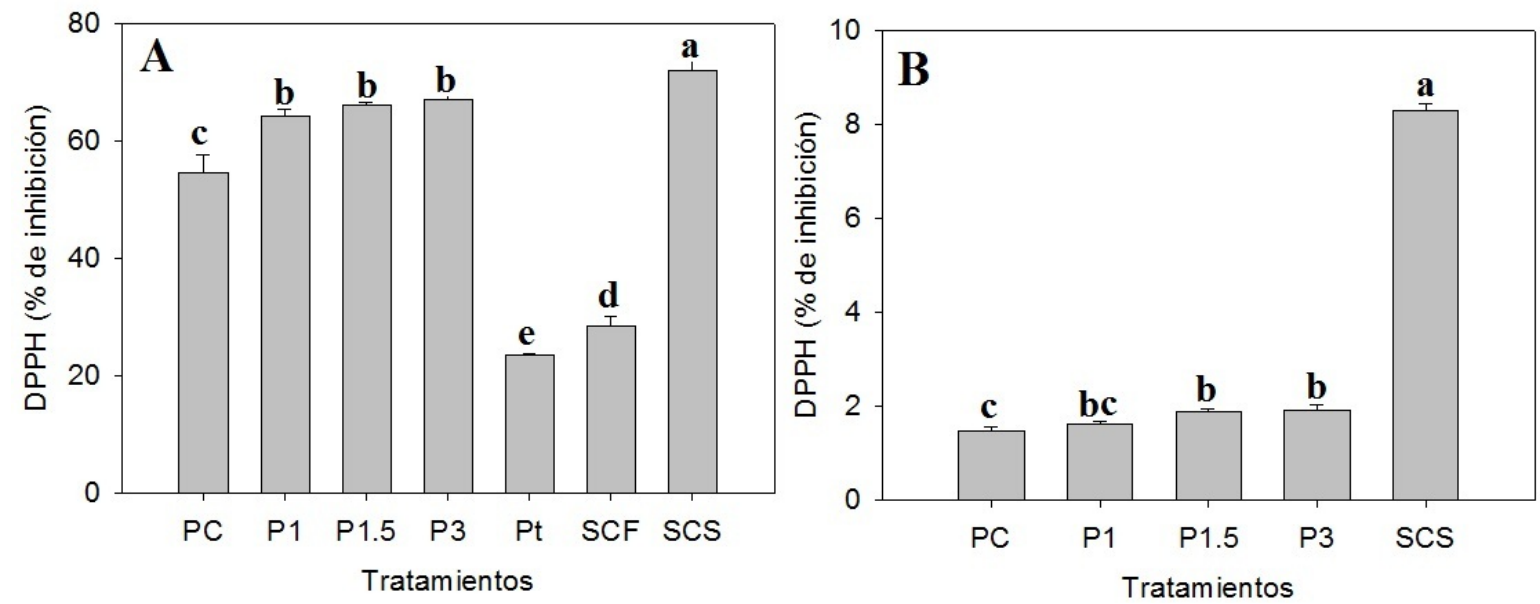

Figura 2. Inhibición del radical DPPH. A: Semana 0 de procesamiento; B: Semana 24 de almacenamiento. Diferente literal (abcde) entre columnas indica diferencia significativa (Tukey $\mathrm{P} \leq 0.05$ ). $\mathrm{PC}=$ Extracto pasta control; $\mathrm{P} 1=$ Extracto de pasta adicionado con $1 \%$ de semilla y cáscara en polvo; P1.5= Extracto de pasta adicionado con $1.5 \%$; P3= Extracto de pasta adicionado con $3 \%$; Pt= Extracto de pulpa de tomate; $\mathrm{SCF}=$ Extracto de semilla y cáscara fresca; $\mathrm{SCS}=$ Extracto de semilla y cáscara seca.

Figure 2. DPPH radical inhibition. A: Week 0 of processing; B: Week 24 storage. Different literal (abcde) means statistically significant difference between treatments (Tukey $\mathrm{p} \leq 0.05$ ). $\mathrm{PC}=$ Control tomato paste extract; $\mathrm{P} 1=$ Tomato paste extract added with $1 \%$ seed and shell powder; P1.5 = Tomato paste extract added with 1.5\%; P3 = Tomato paste extract added with 3\%; Pt = Tomato pulp extract; SCF $=$ Fresh seed and shell extract; $\mathrm{SCS}=$ Seed extract and dried husk. 
Tabla 1. Capacidad antioxidante mediante TEAC y ORAC en 24 semanas de almacenamiento.

Table 1. Antioxidant capacity through TEAC and ORAC in 24 weeks of storage.

\begin{tabular}{lcc}
\hline TRATAMIENTOS & TEAC $(\boldsymbol{\mu M}$ ET/g ps $)$ & ORAC $(\boldsymbol{\mu M}$ ET/g ps \\
\hline PC & $2.547 \pm 0.04 \mathrm{~d}$ & $12.032 \pm 0.14 \mathrm{c}$ \\
P1 & $2.821 \pm 0.02 \mathrm{~d}$ & $13.908 \pm 0.42 \mathrm{bc}$ \\
\hline P1.5 & $4.145 \pm 0.02 \mathrm{c}$ & $17.881 \pm 1.31 \mathrm{bc}$ \\
\hline P3 & $7.241 \pm 0.03 \mathrm{~b}$ & $21.526 \pm 3.45 \mathrm{~b}$ \\
SCS & $14.722 \pm 0.50 \mathrm{a}$ & $53.693 \pm 0.41 \mathrm{a}$ \\
\hline
\end{tabular}

Diferente literal (abcde) dentro de cada columna indica diferencia significativa (Tukey $\mathrm{p} \leq 0.05$ ). $\mathrm{PC}=$ Extracto pasta control; $\mathrm{P} 1=$ Extracto de pasta adicionado con $1 \%$ de semilla y cáscara en polvo; P1.5= Extracto de pasta adicionado con $1.5 \%$; P3= Extracto de pasta adicionado con $3 \%$; $\mathrm{SCS}=$ Extracto de semilla y cáscara seca.

Different literal (abcd) means statistically significant difference between treatments (Tukey $\mathrm{p} \leq 0.05$ ). PC $=$ Control tomato paste extract; P1 = Tomato paste extract added with $1 \%$ seed and shell powder; P1.5 = Tomato paste extract added with 1.5\%; P3 = Tomato paste extract added with 3\%; Pt = Tomato pulp extract; SCF = Fresh seed and shell extract; $S C S=$ Seed extract and dried husk.

rencia significativa $(p>0.05)$ con $P 1$, sin embargo, sí hubo diferencia ( $p \leq 0.05)$ con P1.5 y P3. En las pastas de tomate, P3 presentó el valor de TEAC mayor $(7.241 \pm 0.03 \mu \mathrm{M}$ TE / g) y el comportamiento mostró que el aumento en el contenido de semilla y cáscara de tomate aumentó el valor de TEAC. Los resultados obtenidos en PC y P1 son ligeramente menores a lo reportado por Katırcl et al. (2018) en pasta de tomate comercial $(6.16 \mu \mathrm{M}$ TE / g), pero mayor a pasta de tomate casera (2.67 M TE / g); También Capanoglu et al. (2008) comparó la capacidad antioxidante mediante ABTS de la pasta de tomate en cada etapa del proceso de elaboración y obtuvo $39.88 \pm 3.85 \mu \mathrm{M} \mathrm{TE} / \mathrm{g}$ y Thomas et al. (2017) obtuvieron 5.764 $\pm 3.85 \mu \mathrm{M}$ TE / g. Lo anterior se atribuyó a las condiciones de almacenamiento y tiempo de exposición a la iluminación. Resultados similares fueron reportados por Pokorný et al. (2004), donde la pasteurización y el almacenamiento causaron pardeamiento acompañado de un aumento en la intensidad del color de los vegetales como resultado de la oxidación de los compuestos fenólicos a sus respectivas quinonas. Además, señalaron que, en la composición del tomate, en comparación con otros antioxidantes, incluidos otros carotenoides, el licopeno es relativamente estable, pero se puede perder como resultado de la degradación térmica y la oxidación, se degrada fácilmente por exposición al aire, esto puede ser evidente por una pérdida de enrojecimiento en los productos.

La capacidad antioxidante de los tratamientos utilizando la metodología ORAC fue mayor a la utilizada por TEAC, con una tendencia similar, debido a que ORAC permite analizar la capacidad antioxidante de alimentos o bebidas de una naturaleza más compleja (Zulueta et al. (2009). El tratamiento SCS (53.693 $\pm 0.41 \mu \mathrm{M}$ ET / g) resultó menor a lo reportado por Perea-Dominguez et al. (2018) realizado a subproductos de tomate, que se liofilizaron para conservar la muestra, lo que provocó una mayor conservación de los compuestos antioxidantes.

Adicionalmente, se establecieron coeficientes de correlación de Pearson entre los compuestos fenólicos y la capacidad antioxidante. En la tabla 2 se puede observar una correlación lineal positiva fuerte entre los compuestos fenólicos y la actividad antioxidante con valor de R superior a 0.9. Lo cual indica que, a mayor concentración de compuestos fenólicos, la capacidad antioxidante del alimento es más elevada. Se ha reportado que no todos los compuestos que se extraen, pertenecen al grupo fenólico, y que además pueden tener la capacidad de actuar contra un radical libre en una metodología para determinar la actividad antioxidante (Sagdic et al., 2011). Estos resultados concuerdan con los reportados por Valdez-Morales et al. (2014) quienes reportan correlaciones fuertes con un valor de $\mathrm{R}$ superior a 0.8 entre compuestos fenólicos y actividad antioxidante.

Tabla 2. Coeficientes de correlación de Pearson entre el contenido de compuestos fenólicos y la capacidad antioxidante.

Table 2. Pearson's correlation coefficients between phenolic compound content and antioxidant capacity.

\begin{tabular}{lcccc}
\hline & Fenoles & DPPH & ORAC & TEAC \\
\hline Fenoles & 1 & & & \\
DPPH & 0.990 & 1 & & \\
ORAC & 0.984 & 0.966 & 1 & \\
TEAC & 0.963 & 0.949 & 0.974 & 1 \\
\hline
\end{tabular}

\section{CONCLUSIONES}

A partir de los resultados, se concluyó que la cáscara y semilla deshidratada del tomate son ricas en compuestos fenólicos con alta actividad antioxidante, y estos se unen principalmente a los componentes estructurales de las paredes celulares. Cuando se determina la actividad antioxidante de un alimento se buscan técnicas que se complementen, por lo que se prefiere evaluar varios métodos en lugar de un enfoque unidimensional, ya que algunas técnicas como ORAC utiliza radicales libres de importancia biológica y se basa en la transferencia de átomos de hidrógeno, mientras que DPPH y TEAC se basan en la transferencia de electrones en radicales sintéticos. La valorización de la semilla y cáscara obtenida de un proceso industrial del tomate, podría generar ingresos adicionales para la industria del tomate y reducir el problema de eliminación de desechos. Los resultados de este estudio brindan a los productores de tomate y empresarios información que permite denotar que, agregar semilla y cáscara de tomate a una pasta, mejora el contenido de fenoles y antioxidantes del alimento, sin embargo, el procesamiento tradicional y secado convencional utilizado en los tratamientos provocó disminución de la cantidad de fenoles totales y capacidad antioxidante con respecto a lo reportado en muestras liofilizadas. Es por lo anterior que la industria alimenticia debe decidir sobre el costo-beneficio del producto que desean obtener. El almacenamiento en condiciones normales provocó una disminución de los anti- 
oxidantes presentes, por lo que es indispensable adecuar el almacenamiento en condiciones controladas que permitan una mejor conservación de los antioxidantes.

\section{AGRADECIMIENTOS}

Se agradece a Tecnológico Nacional de México, especialmente al Instituto Tecnológico Superior de Guasave por el recurso económico y por brindar las instalaciones para el desarrollo del proyecto. Gracias al Clúster Hortaliceros Unidos de Sinaloa por su colaboración en la investigación.

\section{REFERENCIAS}

Abouel-Yazeed, A. M., Abou-Tor, E. S. y Boriy, E. G. 2019.Utilization of husk tomato fruit seeds powder in some foodstuff. J. Food and Dairy Sci., Mansoura Univ., Vol. 10(8): 281- 288.

Brand-Williams, W., Cuvelier, M. E., y Berset, C. 1995. Use of a free radical method to evaluate antioxidant activity. LWT- J. Food Sci. Technol. 28: 25-30.

Büyükbay, E. O., Sayili, M., y Uzunoz, M. 2009. The relationship between socio-economic characteristics and sauce consumption of consumers: case of Tokat province. Electronic Journal of Food Technologies, 4(1), 1-7.

Capanoglu, E., Beekwilder, J., Boyacioglu, D., Hall, R., y de Vos, R. 2008. Changes in Antioxidant and Metabolite Profiles during Production of Tomato Paste. Journal of Agricultural and Food Chemistry, 56(3), 964-973. doi:10.1021/jf072990e

Dorais, M., Ehret, D. L., y Papadopoulos, A. P. 2008. Tomato (Solanum lycopersicum) health components: from the seed to the consumer. Phytochemistry Reviews, 7(2): 231-250. doi:10.1007/s11101-007-9085-x

Elbadrawy, E., y Sello, A. 2016. Evaluation of nutritional value and antioxidant activity of tomato peel extracts. Arabian Journal of Chemistry, 9, S1010-S1018. doi:10.1016/j. arabjc.2011.11.011

Karadag, A., Ozcelik, B., y Saner, S. (2009). Review of methods to determine antioxidant capacities. Food analytical methods, 2(1): 41-60.

Katırcı, N., Işık, N., Güpür, Ç., Ozge Guler, H., Gursoy, O., y Yilmaz, Y. 2018. Differences in antioxidant activity, total phenolic and flavonoid contents of commercial and homemade tomato pastes. Journal of the Saudi Society of Agricultural Sciences. doi:10.1016/j.jssas.2018.11.003

Kedare, S. B., y Singh, R. P. 2011. Genesis and development of DPPH method of antioxidant assay. Journal of Food Science and Technology, 48(4): 412-422. doi:10.1007/s13197-0110251-1

Navarro-González, I., García-Valverde, V., García-Alonso, J., y Periago, M. J. (2011). Chemical profile, functional and antioxidant properties of tomato peel fiber. Food Research International, 44(5): 1528-1535. doi:10.1016/j. foodres.2011.04.005

Pellegrini N., Re, R., Min, Y., y Rice-Evans, C. 1999. Screening of dietary carotenoids and carotenoid-rich fruit extracts for antioxidant activities applying 2, 2'-azinobis (3-ethylenebenzothiazoline-6-sulfonic acid radical cation decolorization assay. Methods Enzymol. 299: 379-389.
Perea-Domínguez, X. P., Hernández-Gastelum, L. Z., OlivasOlguin, H. R., Espinosa-Alonso, L. G., Valdez-Morales, M., y Medina-Godoy, S. 2018. Phenolic composition of tomato varieties and an industrial tomato by-product: free, conjugated and bound phenolics and antioxidant activity. Journal of Food Science and Technology, 55(9): 3453-3461. doi:10.1007/s13197-018-3269-9.

Pokorný, J., Schmidt, Š., y Nguyen, H. T. T. 2004. Functionality changes of natural antioxidants during food processing and storage. Czech Journal of Food Sciences, 22: 80-83.

Sagdic, O., Ozturk, I., Ozkan, G., Yetim, H., Ekici, L., y Yilmaz, M. T. 2011. RP-HPLC-DAD analysis of phenolic compounds in pomace extracts from five grape cultivars: Evaluation of their antioxidant, antiradical and antifungal activities in orange and apple juices. Food Chemistry, 126(4): 1749-1758.

Sarkar, A., y Kaul, P. 2014. Evaluation of Tomato Processing ByProducts: A Comparative Study in a Pilot Scale Setup. Journal of Food Process Engineering, 37(3): 299-307. doi:10.1111/ jfpe. 12086

Secretaría de Agricultura, Ganadería, Desarrollo Rural, Pesca y Alimentación (SAGARPA). 2017. Planeación Agrícola Nacional 2017-2030. https://www.gob.mx/cms/uploads/ attachment/file/257077/Potencial-Jitomate.pdf

Shivashankara K. S, S. Isobe, M. I. Al-Haq, M. Takenaka, y T. Shiina. 2004. Fruit antioxidant activity, ascorbic acid, total phenol, quercetin, and carotene of Irwin mango fruits stored at low temperature after high electric field pretreatment. J. Agric. Food Chem. 52: 1281-1286.

Singleton V. L. y J. A. Rossi Jr. 1965. Colorimetry of total phenolics with phosphomolybdic-phosphotungstic acid reagents. Am J Enol Vitic. 16: 144-158.

Valdez-Morales, M., Espinosa-Alonso, L. G., Espinoza-Torres, L. C., Delgado-Vargas, F., y Medina-Godoy, S. 2014. Phenolic Content and Antioxidant and Antimutagenic Activities in Tomato Peel, Seeds, and Byproducts. Journal of Agricultural and Food Chemistry, 62(23): 5281-5289. doi:10.1021/ jf5012374

Vallverdú-Queralt, A., Medina-Remón, A., Casals-Ribes, I., Andres-Lacueva, C., Waterhouse, A. L., y Lamuela-Raventos, R. M. 2012. Effect of tomato industrial processing on phenolic profile and hydrophilic antioxidant capacity. LWT Food Science and Technology, 47(1): 154-160. doi:10.1016/j. Iwt.2011.12.020.

Vinha, A. F., Alves, R. C., Barreira, S. V., Castro, A., Costa, A. S., y Oliveira, M. B. P. 2014. Effect of peel and seed removal on the nutritional value and antioxidant activity of tomato (Lycopersicon esculentum L.) fruits. LWT-Food Science and Technology, 55(1): 197-202.

Zhang, J., Chu, P. C., Chao, C. y Chen, J. 2011. Effect of three cooking methods on nutrient components and antioxidant capacities of bamboo shoot. J Zhejiang Univ-Sci B (Biomed y Biotechnol)

Zulueta, A., Esteve, M. J., y Frígola, A. 2009. ORAC and TEAC assays comparison to measure the antioxidant capacity of food products. Food Chemistry, 114(1): 310-316. 